\title{
Modelling the Social Buffering Hypothesis in an Artificial Life Environment
}

\author{
Imran Khan $^{1}$, Matthew Lewis ${ }^{2}$ and Lola Cañamero ${ }^{3}$ \\ $\left\{\right.$ i.khan9 ${ }^{1} \mid$ m.lewis4 ${ }^{2} \mid$ l.canamero $\left.^{3}\right\}$ @ herts.ac.uk
}

\begin{abstract}
In social species, individuals who form social bonds have been found to live longer, healthier lives. One hypothesised reason for this effect is that social support, mediated by oxytocin, "buffers" responses to stress in a number of ways, and is considered an important process of adaptation that facilitates long-term wellbeing in changing, stressful conditions. Using an artificial life model, we have investigated the role of one hypothesised stress-reducing effect of social support on the survival and social interactions of agents in a small society. We have investigated this effect using different types of social bonds and bond partner combinations across environmentally-challenging conditions. Our results have found that stress reduction through social support benefits the survival of agents with social bonds, and that this effect often extends to the wider society. We have also found that this effect is significantly affected by environmental and social contexts. Our findings suggest that these "social buffering" effects may not be universal, but dependent upon the degree of environmental challenges, the quality of affective relationships and the wider social context.
\end{abstract}

\section{Introduction}

Positive social relationships play a significant role in the wellbeing of individuals in social species. For many species, individuals who maintain close social bonds have longer, healthier lives and reduced stress levels (DeVries et al., 2003; Holt-Lunstad et al., 2010; Levine, 2000). One way in which these relationships are hypothesised to provide these beneficial effects is through the "social buffering" phenomenon (Hennessy et al., 2009; Kikusui et al., 2006), which posits that social support provides individuals with a physiological and psychological "safety cushion" (Wittig et al., 2016) during stressful events. This has also been seen to reduce and adapt stress responses to future stressful situations (Wittig et al., 2016; Heinrichs et al., 2003), suggesting an anxiolytic effect of social support-likely as a result of oxytocin's adaptive effects on stress-related physiological systems (Heinrichs et al., 2003).

This physiological adaptation through the social environment underpins the concept of social allostasis (Schulkin et al., 2004; Sterling, 2004), which describes the adapta- tion of the stability-seeking homeostatic mechanism (Cannon, 1929) over an individual's lifetime, through internal, external, and social contexts. Our current work (Khan et al., 2019) is concerned with developing an adaptive, embodied model of social allostasis to facilitate long-term viability (Ashby, 1954) of (artificial) social agents across dynamic physical and social conditions.

Recent work from the field has also investigated the role of social interactions on the performance of agent models, such as the effects on cooperation (Andras, 2016; Rajagopalan et al., 2019), including affect-based approaches (Antunes and Magnenat-Thalmann, 2016; Santos et al., 2011); social learning (Marriott and Chebib, 2014; Jolley et al., 2016), and the evolution of social dynamics (Covert III et al., 2014; Miramontes et al., 2016). Though some work has looked at allostatic(-type) models for adaptation (Vouloutsi et al., 2013; Moulin-Frier et al., 2017; Lones et al., 2017), a model focusing on mechanisms of social allostasis is yet to be investigated.

Building on the social buffering hypothesis, we investigate the role of the presence of affective social bond partners on the wellbeing and social interactions of a small society across dynamic environmental conditions. Using a simulated social model of agents, we investigate these effects for two different types of affective social bonds-one where the bond strength between bond partners remains fixed, and one where the bond strength is strengthened and weakened through social interactions-across a number of different bond partner combinations related to social rank. We hypothesise that affective social bonds will provide survivalrelated (viability) benefits for bond partners across dynamic physical conditions, and that this improved survival will be facilitated by a reduction in stress and socio-negative interactions between bond partners. We present and discuss our experiments to test this hypothesis in this paper. Our results show some support for our hypothesis, and that the stressreducing effects of social support is affected by the social and environmental contexts, as well as the type of affective bond between agents. 


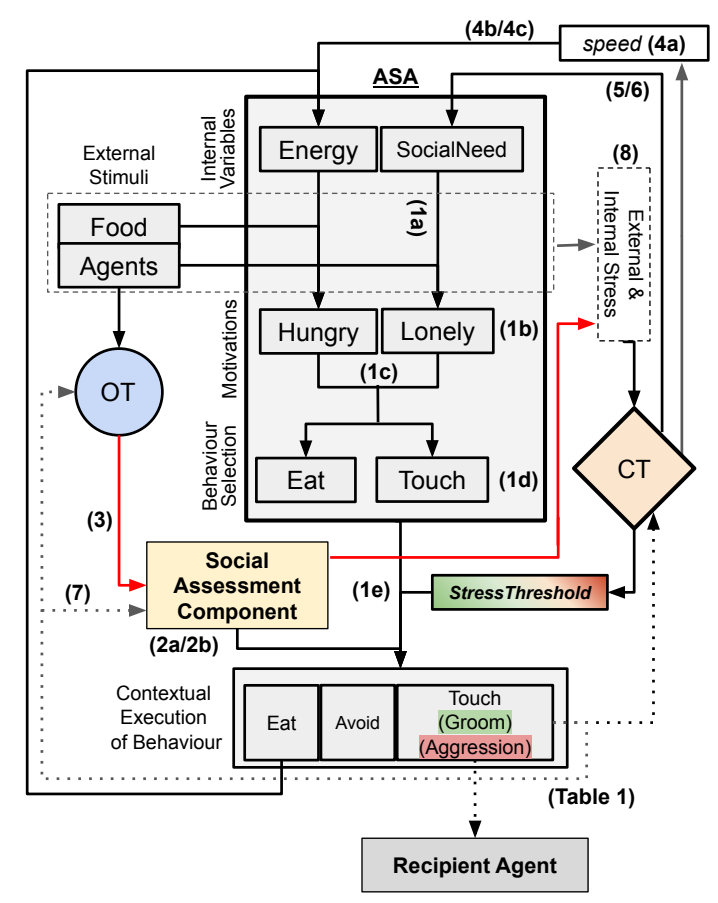

Figure 1: Overview of the agent model used in this investigation. A more in-depth view of the Social Assessment Component is seen in Figure 2. Red lines indicate the modelling of the "social buffering" effect investigated in this paper. Numbers in brackets denote corresponding equation. Dotted lines denote contextual effects on hormones (Table 1). Green highlights behaviour when agents are not stressed, red highlights behaviour when agents are stressed. ASA = Action-Selection Architecture. OT $=$ Oxytocin. $\mathrm{CT}=$ Cortisol.

\section{Agent Model}

\section{Action-Selection Architecture}

Using the long-standing approach from our research group (Cañamero, 1997; Lones et al., 2017; Lewis and Cañamero, 2019), agent behaviour is driven by a homeostaticallycontrolled decision-making architecture, simply called the Action-Selection Architecture (ASA). The ASA selects behaviours in order to satisfy one of two physiological needs (Table 1). The first, Energy, is a physical need and a survival-critical variable: if it drops to its lower bound, the agent will "die". The second, SocialNeed, is a non-critical need for social contact (i.e. agents can die of Hunger but not Loneliness) though it still drives error-correcting behaviours. These internal variables experience a small decay at each time step. The goal of this homeostatically-controlled model is to maintain stability by keeping these values as close to their ideal values as possible through one of two deficitcorrecting behaviours: Eat or Touch.

The ASA runs at each time step and works as follows:

\begin{tabular}{|c|c|c|c|c|c|}
\hline $\begin{array}{c}\text { Internal } \\
\text { Variables } \\
\text { P }\end{array}$ & Motivation & Behaviour & Stimuli & $\begin{array}{c}\text { Phys. } \\
\text { Effect } \\
\text { m }\end{array}$ & $\begin{array}{c}\text { Phys. } \\
\text { Effect } \\
\text { i }\end{array}$ \\
\hline Energy & Hungry & Eat & Food & + Energy & - \\
\hline SocialNeed & Lonely & $\begin{array}{c}\text { Touch } \\
\text { (Groom) }\end{array}$ & Agent & $\begin{array}{c}+ \text { SocialNeed } \\
+ \text { OT }\end{array}$ & $\begin{array}{c}-\mathrm{CT} \\
+\mathrm{OT}\end{array}$ \\
\hline SocialNeed & Lonely & $\begin{array}{c}\text { Touch } \\
\text { (Aggression) }\end{array}$ & Agent & $\begin{array}{c}+ \text { SocialNeed } \\
-C T\end{array}$ & $+\mathrm{CT}$ \\
\hline
\end{tabular}

Table 1: Relationship between each internal variable, motivations, behaviours and stimuli required to perform each behaviour. "Phys. Effect" = Physiological effects on performing agent (A) and recipient agent (i). OT $=$ Oxytocin. $\mathrm{CT}=$ Cortisol.

The error of each internal variable $i$ is calculated as the difference between its ideal and current value (Eq. 1a). This error is then combined with the perceived availability of relevant stimuli $\left(c_{i}\right)$ to calculate the "urgency" of each motivation (Eq. 1b). The motivation with the highest value is selected as the winning motivation (Eq. 1c). The behaviour with the largest physiological effect $\left(p_{j i}\right)$ for the winning motivation is selected as the winning behaviour. As each motivation corresponds to a single behaviour, the resulting behaviour will either be Eat when the winning motivation is Hungry, or Touch when the winning motivation is Lonely (Eq. 1d,1e). Therefore:

$$
\begin{array}{r}
d_{i}=\text { ideal }_{i}-\text { current }_{i} \\
m_{i}=d_{i}+\left(d_{i} \times c_{i}\right) \\
m_{\text {winner }}=\max \left(m_{\text {Hungry }}, m_{\text {Lonely }}\right) \\
b_{i}=m_{i} \times p_{j i} \\
b_{\text {winner }}=\max \left(b_{\text {Eat }}, b_{\text {Touch }}\right)
\end{array}
$$

This winning behaviour then drives the agent towards relevant resources in order to satisfy their current motivation. Further details can be seen in Table 1.

\section{Social Assessment Component}

The Social Assessment Component (SAC) is an extension to the ASA (Khan et al., 2019), which introduced an additional step before behaviour execution by accounting for the social context-in this case, social rank differences and affective bond with others. Similar to the approach used in DomWorld (Hemelrijk et al., 2017), social rank is a value representing an agent's hierarchical status within the society, and is associated with priority access to food and preference in being chosen as a grooming partner. An affective bond is a flag that represents a mutually-positive relationship between two agents. When the ASA calculates a winning behaviour, the SAC calculates a value (AgentVal) for each perceived agent ( $i$ ) which determines (a) approach/avoid decisions when the winning behaviour is to Eat, and (b) partner selection when the winning behaviour is Touch. This 


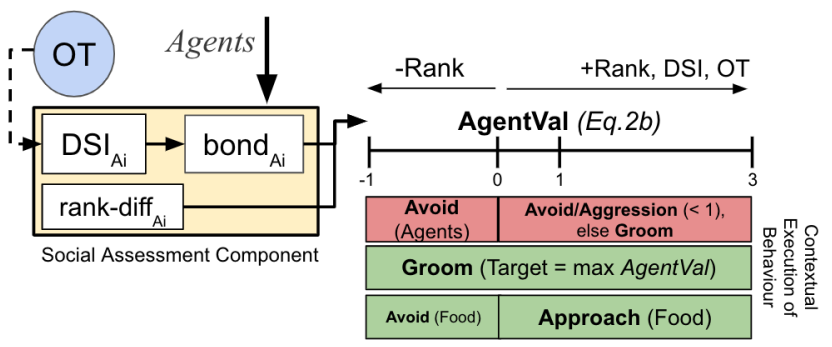

Figure 2: Illustration of the Social Assessment Component calculation (left) and the contextual social behaviours based on the AgentVal calculation (right). Red = behaviours when agents are stressed $(\mathrm{CT} \geq$ StressThreshold), green $=$ behaviours when agents are not stressed. $O T=$ Oxytocin. DSI $=$ Dyadic Strength Index. rank-diff $A_{i}$ is the normalised difference in rank between the two agents (Equation 2).

value is calculated using the difference in social rank between agents $A$ and $i$, and the presence (and quality) of an affective bond. A socially-influenced hormone of oxytocin (OT) is then used to modulate the valence of affective bonds in this calculation:

$$
\text { rank-diff }{ }_{A i}=\operatorname{rank}_{i}-\operatorname{rank}_{A}
$$

$$
\text { AgentVal }_{i}={\operatorname{rank}-\operatorname{diff}_{A i}}+\left(\text { bond }_{A i} \times \operatorname{DSI}_{A i} \times O T_{A}\right)
$$

where rank-diff is a normalised value between -1 and +1 (in 0.25 increments) denoting the difference in social rank between agents $A$ and $i$. A negative value corresponds to a perceived agent being higher-ranked, and vice-versa. The bond ${ }_{A i}$ flag denotes if an affective bond exists between Agent $A$ and $i$ ( 1 if bond exists, else 0 ). The strength of this affective bond is denoted with the Dyadic Strength Index (DSI), in the range 0 to 2. Finally, OT denotes the level of oxytocin in an agent's physiology, between 0 and 1 . Agents turn away from (avoid) occupied food and other agents at a small angle when AgentVal $<0$. Finally, agents are driven to Groom with the agent returning the largest AgentVal.

\section{Hormonal Effects}

We include two hormones in our agent model. The first, oxytocin (OT), is a modulatory hormone, released in response to performing or receiving positive social interaction (Grooming). OT is released in both the initiator and recipient of the prosocial behaviour as a function of the intensity $\left(\mathrm{b}_{\text {Touch }}\right)$ of the Touch behaviour:

$$
O T_{t}=O T_{t-1}+\left(1 \times \mathrm{b}_{\text {Touch }}\right)
$$

OT experiences a small decay (-0.005) at each time step. In the SAC calculation, OT is used to modulate the valence of affective bond partners (Eq. 2): an abstraction of OT's effects on preferential attention towards affective partners (Taylor, 2006).

The second hormone, cortisol (CT) is a stress-related hormone with a number of modulatory effects. Firstly, it modulates the speed at which an agent moves. This increased speed depletes Energy at an increased rate. Therefore:

$$
\begin{array}{r}
\text { speed }_{t}=\text { speed }_{\text {default }} \times(1+(C T \times c) \\
\text { EnergyCost }_{t}=\text { EnergyCost }_{\text {default }} \times\left(2 \times \text { speed }_{t}\right) \\
\text { Energy }_{t}=\text { Energy }_{t-1}-\text { EnergyCost }
\end{array}
$$

The second effect is that it increases the intensity of tactile behaviours being performed. The more CT in an agent's system, the more "intense" the strength of the tactile behaviour:

$$
\text { TactileIntensity }=b_{\text {touch }} \times \mathrm{CT}
$$

For the performing agent, this value determines the rate of their internal SocialNeed satisfaction:

$$
\text { SocialNeed }_{t}=\text { SocialNeed }_{t-1}+(\text { TactileIntensity } \times c)
$$

CT is reduced by the value of (TactileIntensity $\times c$ ) in the recipient agent during Grooming, but increased by the same amount when receiving Aggression. At the same time, the strength of a bond (DSI) is also increased or decreased as a function of TactileIntensity, depending on the behaviour:

$$
D S I_{A i}= \begin{cases}D S I_{A i}+\text { TactileIntensity } \times c & \text { if Groom } \\ D S I_{A i}-\text { TactileIntensity } \times c & \text { if Aggression }\end{cases}
$$

where $c$ is a constant to regulate values. The rate at which cortisol is secreted or reduced is a function of physiological (internal) stress (the deficits of internal variables), and psychological (external/social) stress (related to difficulties in satisfying internal deficits). Specifically:

$$
\text { CTReleaseRate }_{A}=\frac{1}{2}\left(\sum_{i \in P} d_{i}-\sum_{i \in S} \hat{S}_{i}\right) \times c
$$

where $\sum d_{i}$ is the sum of errors of both internal variables $(P), \sum \hat{S}_{i}$ is the sum of perceived "available" resources $(S)$, and $c$ is a fixed constant. Availability of resources (food or agents) are those which an agent perceives it can access. Accessibility of resources is determined through the AgentVal calculation:

$$
\begin{array}{r}
\sum d_{i}=d_{\text {Energy }}+d_{\text {SocialNeed }} \\
\sum \hat{S}_{i}=\hat{S}_{\text {Agents }}+\hat{S}_{\mathrm{Food}}
\end{array}
$$


where $\left(\hat{S}_{\text {agents }}=C_{\text {Agents }} \times(1-\right.$ AgentVal $\left.)\right)$ and $\left(\hat{S}_{\text {Food }}=1\right.$ when AgentVal $\geq 0$, else 0 ).

As OT and (the presence and quality of) affective bonds are included in the assessment of "available resources" when calculating CTReleaseRate, it offsets the stress response associated with internal and external stress, therefore "buffering" and reducing (the release rate of) CT.

Finally, each agent has a fixed internal tolerance to the amount of CT it can withstand before it becomes "stressed". For this investigation, this StressThreshold is set to 0.5: when CT levels exceed this threshold, agents are stressed and adapt their social behaviours accordingly by either increased avoidance or Aggression towards others (Figure 2).

\section{Agent Perception and Behaviours}

Vision System: Each agent has a fixed field-of-vision of length 20 units with an angle of 80 degrees (+/- 40 degrees) and can only perceive resources that fall within this range.

Movement: Random wandering constitutes an appetitive behaviour and is performed in absence of any relevant stimuli. Agents have a default wandering speed $(0.5$ units per time step), that is modulated by the amount of cortisol in their physiology (Equation 4). When agents perceive a stimuli that satisfies its current motivation, they focus on that resource and move towards it. Agents turn at a small angle $\left(90^{\circ} \times C T\right)$ from agents where AgentVal $<0$.

Eat: When agents reach a food resource and look to satisfy their Hunger motivation, they stop and take "bites" of the resource, satisfying their internal Energy need at a fixed rate $(+0.01)$ until their drive has been satisfied.

Touch (Groom and Aggression): Touch encapsulates both a socio-positive (Groom) and socio-negative behaviour (Aggression), dependent upon the stressed state of the agent and its social relationship with the other agent (via the AgentVal). In both cases, this behaviour takes place within a single time step. Recipient agents are "pushed" a short distance, relative to the intensity of tactile contact received. Groom and Aggression have opposite effects on CT (Table 1 ). The strength of a bond (DSI) is strengthened and weakened through Aggression (Equation 7).

\section{Experiments \& Results}

\section{Methodology}

Experiments were conducted using the NetLogo platform (Wilensky, 1999) v5.3.1. Our model used a society of six agents (A1 to A6), with all agents holding a different hierarchical rank (Figure 3 ) and endowed with the action-selection model described in the Agent Model section. Experimental conditions were defined in terms of the presence and type of affective bonds, the affective bond combination, and the type of environment related to food availability, We ran 20 simulations for each experimental condition, and each simulation ran for 15,000 time steps.

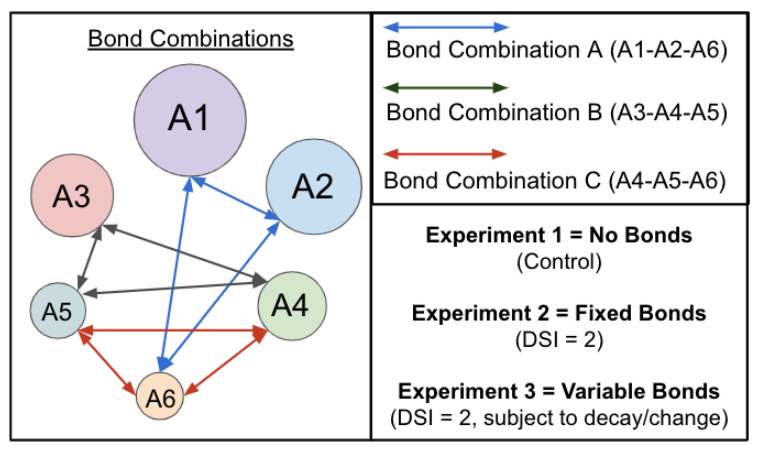

Figure 3: Illustration of the three different bonded conditions used in Experiment $2 \& 3$ (left) and brief description of bond types. Number represents agents' dominance rank (A1 = highest, $\mathrm{A} 6=$ lowest $)$.

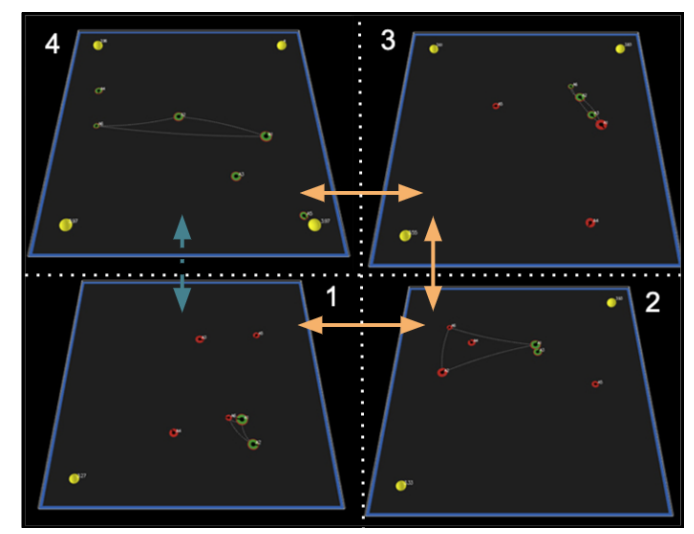

Figure 4: Screenshots of the different world conditions. Yellow discs represent food resources. Agents are flat "doughtnut" (green and red) shapes. Static worlds are seen in the top left. Orange arrows show changes in Seasonal conditions. Blue arrows show changes in the Extreme conditions.

In terms of the types of affective bonds, Experiment 1 had all agents unbonded and served as our control. In Experiment 2 , affective bond strength remained fixed throughout the experiment (DSI = 2). In Experiment 3, affective bond strength was variable, with the bond strength (DSI) subject to decay ( $\mu=0.9997)$ and changes as a function of social interactions (Equation 7).

In Experiments 2 \& 3, we investigated three different affective bond partner combinations (Figure 3 ). In each condition, three agents were bonded and three were unbonded. Bond combinations related to the social rank of agents. Bond Condition A had A1-A2-A6 with affective bonds between them, Bond Condition B had A3-A4-A5 with bonds, and Bond Condition $\mathrm{C}$ had A4-A5-A6 with bonds.

Experiments were performed across three world conditions related to food availability. The first was a Static 
world where food availability remained constant (Figure 4, top left). Seasonal was a dynamic environment with food changes every 1000 time steps (after an initial static phase of 2000 time steps, Figure 4, orange arrows), steadily changing from 4 foods (Phase 1) to 1 food (Phase 4). The Extreme environment had more significant food change during these same phases (4 foods in Phase 1, 1 food in Phase 2; Figure 4 , blue arrows).

In terms of metrics, we measured results across three viability-related (Ashby, 1954) measures: Life Length (LL), describing the length of time an agent or group survives as a percentage of total simulation run time (from 0\%$100 \%$ ); Mean Comfort (MC), describing the mean value of the two homeostatically-controlled variables (between 01 ); and Physiological Wellbeing (PW), describing the homogenity of the satisfaction of these two internal variables (between 0-1). We also measured mean hormone levels and the distribution of social behaviours. We complemented our quantitative results with qualitative analysis of the experiments. Statistical significance testing was performed using one-way ANOVA testing.

\section{Results: Viability Indicators}

Experiment 1 (Control): As expected, viability performance of the overall society was greatest in the Static environments (LL: 33\%, PW: 0.75). The results from the two dynamic world conditions were similar for both of these viability indicators (LL: 22\%, PW: 0.49, 0.51 for Seasonal and Extreme conditions respectively) (Table 2).

Experiment 2: Like control, viability was greatest in the Static world condition (LL: 49\%-53\%, PW: 0.95), with bonded (B) groups outliving unbonded (UB) agents (B: 5155\%, UB: 46-50\%) and a slightly improved Physiological Wellbeing (+0.15 vs. UB). Viability improvements were also seen in the Seasonal condition (LL: $34 \%-37 \%$, PW: $0.56-$ 0.64 ) with bonded agents (LL: $37 \%-39 \%$, PW: 0.59-0.70) again seeing improved performance vs. UB agents (LL: 31\%-33\%, PW: 0.54-0.61). In Extreme environments, group viability was improved across all bond combinations (LL: 33\%-37\%, PW: 0.65-0.73), with bond agents (LL: 35\%39\%, PW: 0.72-0.79) outperforming unbonded agents (LL: 30\%-39\%, PW: 0.55-0.74) in all but one Bond Condition (C) where there was a non-significant difference between bonded and unbonded viability. All other reported results were statistically-significant $(p<0.01)$ vs. control.

Experiment 3: All bond combinations saw significant increases in viability vs. control. This was most notable in the Seasonal (LL: $+39 \%$ to $+42 \%$, PW: +0.29 ) and Extreme (LL: $+43 \%$ to $+45 \%$, PW: +0.25 ) conditions. Again, bonded agents (LL: 31\%-46\%, PW: 0.50-0.86) showed improved viability compared to unbonded (LL: $27 \%-41 \%$, PW: $0.48-$ 0.79 ), though group survival was lower (LL: $-5.9 \%$ to $-29 \%$ ) vs. fixed bonds. We highlight the largest differences in grey in Table 2. Physiological Wellbeing for all com-

\begin{tabular}{|c|c|c|c|c|}
\hline \multirow[b]{2}{*}{$\begin{array}{c}\text { World } \\
\text { Condition }\end{array}$} & \multicolumn{4}{|c|}{ Life Length (LL) } \\
\hline & Bond & $\begin{array}{l}\text { Control } \\
\text { (1) }\end{array}$ & $\begin{array}{c}\text { Fixed } \\
\text { Bonds } \\
(2)\end{array}$ & $\begin{array}{c}\text { Variable } \\
\text { Bonds } \\
\text { (3) }\end{array}$ \\
\hline Static & $\mathbf{A}$ & $32.8 \%$ & $53.0 \%$ & $43.0 \%$ \\
\hline Static & B & $32.8 \%$ & $49.0 \%$ & $34.8 \%$ \\
\hline Static & $\mathbf{C}$ & $32.8 \%$ & $49.7 \%$ & $46.7 \%$ \\
\hline Seasonal & $\mathbf{A}$ & $21.7 \%$ & $37.3 \%$ & $30.7 \%$ \\
\hline Seasonal & B & $21.7 \%$ & $34.3 \%$ & $30.7 \%$ \\
\hline Seasonal & $\mathbf{C}$ & $21.7 \%$ & $34.6 \%$ & $30.3 \%$ \\
\hline Extreme & $\mathbf{A}$ & $21.6 \%$ & $36.3 \%$ & $36.6 \%$ \\
\hline Extreme & B & $21.6 \%$ & $33.3 \%$ & $31.3 \%$ \\
\hline Extreme & $\mathbf{C}$ & $21.6 \%$ & $36.6 \%$ & $31.0 \%$ \\
\hline $\begin{array}{c}\text { World } \\
\text { Condition }\end{array}$ & Bond & \multicolumn{3}{|c|}{ Physiological Wellbeing (PW) } \\
\hline Static & $\mathbf{A}$ & 0.75 & 0.95 & 0.77 \\
\hline Static & B & 0.75 & 0.95 & 0.79 \\
\hline Static & $\mathbf{C}$ & 0.75 & 0.95 & 0.79 \\
\hline Seasonal & $\mathbf{A}$ & 0.49 & 0.64 & 0.77 \\
\hline Seasonal & B & 0.49 & 0.56 & 0.78 \\
\hline Seasonal & C & 0.49 & 0.60 & 0.77 \\
\hline Extreme & $\mathbf{A}$ & 0.51 & 0.65 & 0.75 \\
\hline Extreme & B & 0.51 & 0.71 & 0.76 \\
\hline Extreme & C & 0.51 & 0.73 & 0.76 \\
\hline
\end{tabular}

Table 2: Viability Indicator results across all experimental conditions, aggregated for all six agents in the society. Differences in bonded and unbonded viability discussed where relevant. Mean Comfort values were non-significant, and have not been included in the analysis.

binations were significantly improved vs. control (+0.03 to +0.29). Despite lower Life Lengths, aggregated Physiological Wellbeing remained consistent for all conditions $(0.75-$ $0.79)$.

Overall, these showed viability improvements for both bonded and unbonded agents when affective bond partners were available for some agents. Comparing experiments, viability was significantly improved for the overall society in experiments with fixed bonds, with smaller viability improvements noted in Experiment 3 vs. control conditions.

\section{Results: Hormone Levels}

Experiment 1: With no affective bonds, we saw that mean CT levels for the group increased as the physical challenge increased (Static: 0.49, Seasonal, 0.68, Extreme, 0.71). OT was not present in these conditions.

Experiment 2 saw lower overall CT levels for bonded and, in some conditions, unbonded agents (Table 3, grey cells) vs. control. CT levels for bonded agents were between $0.28-0.37$ in Static environments, $0.56-0.58$ in the Seasonal environment, and 0.62-0.65 in the Extreme environments. We noted significantly high levels of OT for all conditions (0.82-0.95). We also saw reduced levels of CT in unbonded agents (Static: 0.36-0.42, Seasonal: 0.49-0.60, Extreme: 0.54-0.65). Here, the presence of fixed bonds for three agents reduced the stress of all agents in the society.

Experiment 3 saw comparative CT levels for both bonded and unbonded agents vs. fixed bonds, with some differences (Table 3, dark grey cells). In Static world con- 


\begin{tabular}{|cc|c|ccc|ccc|}
\hline & & $\begin{array}{c}\text { Experiment 1 } \\
\text { (No Bonds) }\end{array}$ & \multicolumn{3}{|c|}{$\begin{array}{c}\text { Experiment 2 } \\
\text { (Fixed Bonds) }\end{array}$} & \multicolumn{3}{c|}{ Experiment 3 } \\
(Variable Bonds)
\end{tabular}

Table 3: Mean oxytocin (OT) and cortisol (CT) levels for bonded (B) and unbonded (UB) agents across all experiments. Results in grey are discussed in this section.

ditions, bonded agents in Bond Conditions $\mathrm{B}$ and $\mathrm{C}$ experienced significantly higher $\mathrm{CT}$ levels vs. unbonded agents $(+0.09$ and +0.19 , respectively). Conversely, we saw lower mean CT levels for bonded agents in Bond Condition A in both Seasonal and Extreme environments vs. fixed bonds. Compared to the other two bond combinations, Bond Condition A experienced the lowest mean CT levels in Seasonal and Extreme world conditions. We also highlight significantly lower OT levels for bonded agents (between 50\%$59 \%, \mathrm{p}<0.001$, Table 3 ) vs. fixed bonds.

Overall, we find lower mean CT levels in both experiments when (both fixed and variable) social bonds are present, compared to conditions with no bonds. In some conditions, we also find stress to be reduced in unbonded agents. We noted significantly reduced OT levels in experiments with variable bonds, with moderately-strong correlations between OT and Life Length (A: $r=0.55, \mathrm{p}=0.032$, B: $r=0.61, p=0.041, C: r=0.67, p<0.001)$.

Our results suggest that the stress-reducing benefits (Table 3 ) and resultant viability (Table 2) associated with social support is dependent upon the type ("fixed" or "variable") of affective social bond.

\section{Results: Social Interactions}

Experiment 1: Due to the increased CT levels from increasing world challenges (Table 3), Aggression increased through the three environments, accounting for $12 \%, 35 \%$, and $39 \%$ of total social interactions. We observed a steady distribution of aggressive encounters in Static environments, and a cyclical distribution of Aggression related to the phases of food availability in Seasonal and Extreme environments. As no bonds were present, no intra-bond social behaviours were measured.

Experiment 2: Intra-bond Grooming increased as the world challenge increased (Table 4). Agents in Bond Condition A showed the highest amount of intra-bond Grooming (63\% to 83\%), while Bond Condition C exhibited the lowest amounts (17\% to 23\%). Intra-bond Aggression was highest in the Seasonal world condition for all bonds: accounting

\begin{tabular}{cccc}
$\begin{array}{c}\text { Bond } \\
\text { Condition }\end{array}$ & $\begin{array}{c}\text { World } \\
\text { Condition }\end{array}$ & $\begin{array}{c}\text { Intra-Bond } \\
\text { Grooming \% }\end{array}$ & $\begin{array}{c}\text { Intra-Bond } \\
\text { Aggression \% }\end{array}$ \\
\hline A & Static & $63 \%$ & $0 \%$ \\
A & Seasonal & $73 \%$ & $10 \%$ \\
A & Extreme & $83 \%$ & $2 \%$ \\
\hline B & Static & $42 \%$ & $0 \%$ \\
B & Seasonal & $45 \%$ & $56 \%$ \\
B & Extreme & $46 \%$ & $8 \%$ \\
\hline C & Static & $17 \%$ & $0 \%$ \\
C & Seasonal & $24 \%$ & $100 \%$ \\
C & Extreme & $23 \%$ & $100 \%$
\end{tabular}

Table 4: Social behaviour of bonded agents in Experiment 2, as a percentage of total Grooming/Aggression performed by bonded agents. Grey results are discussed in this section.

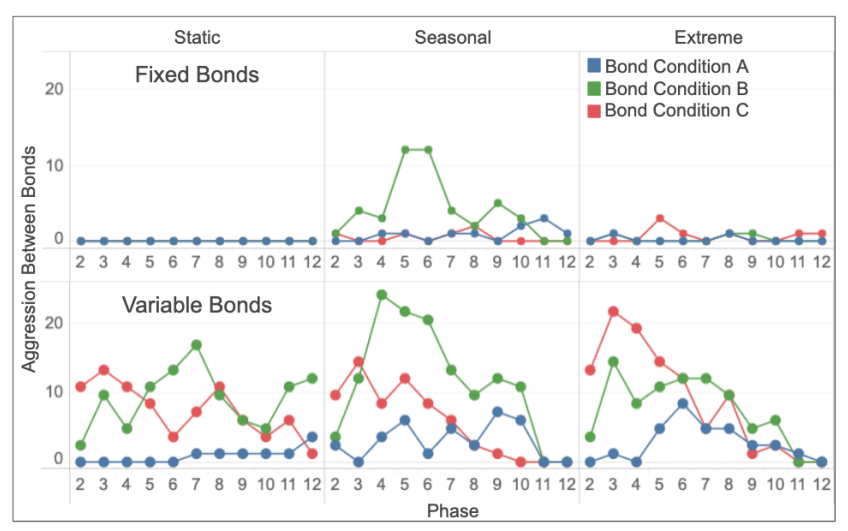

Figure 5: Amount of intra-bond Aggression performed during each phase of food availability (Phase 2-12 only), broken down by Bond Condition (colour). Experiment $2=$ Fixed Bonds (top). Experiment 3 = Variable Bonds (bottom).

for between 10\% (Bond Condition A), and 56\% (Bond Condition B) of all Aggression performed by bonded agents. Despite a significant world challenge, few intra-bond Aggression behaviours were observed in the Extreme environment (Figure 5).

Experiment 3: Intra-bond Grooming was similar vs. Experiment $2(64 \%, 37 \%$ and $15 \%$ for Bond Conditions A, B, and $\mathrm{C}$ respectively). However, intra-bond Aggression saw a significant increase: Bond Condition A saw intra-bond $\mathrm{Ag}$ gression between 21\%-35\% of total Aggression performed by bonded agents; $78 \%-92 \%$ for Bond Condition B, and $100 \%$ in Bond Condition C. In contrast to fixed bond groups, a significant amount of socio-negative behaviours performed by bonded agents were on their own bond partners. In Seasonal environments, Bond Condition B saw significant intrabond Aggression during the phase of worst food availability (Phase 4, 1 food: Figure 5), and maintained high levels of intra-bond Aggression in successive phases. In the Extreme condition, intra-bond Aggression was increased during phases of good food availability (phase 3,5,7), particularly for Bond Conditions B and C (Figure 5). 


\begin{tabular}{cccc}
$\begin{array}{c}\text { Bond } \\
\text { Condition }\end{array}$ & $\begin{array}{c}\text { World } \\
\text { Condition }\end{array}$ & $\begin{array}{c}\text { Intra-Bond } \\
\text { Grooming \% }\end{array}$ & $\begin{array}{c}\text { Intra-Bond } \\
\text { Aggression \% }\end{array}$ \\
\hline A & Static & $64 \%$ & $21 \%$ \\
A & Seasonal & $60 \%$ & $32 \%$ \\
A & Extreme & $60 \%$ & $35 \%$ \\
\hline B & Static & $37 \%$ & $78 \%$ \\
B & Seasonal & $42 \%$ & $91 \%$ \\
B & Extreme & $29 \%$ & $78 \%$ \\
\hline C & Static & $15 \%$ & $100 \%$ \\
C & Seasonal & $17 \%$ & $100 \%$ \\
C & Extreme & $22 \%$ & $100 \%$
\end{tabular}

Table 5: Social behaviour of bonded agents in Experiment 3, as a \% of total Grooming/Aggression performed by bonded agents. Grey results denote notable differences vs. Table 4.

\section{Discussion}

Regardless of the type of affective bond (fixed or variable), we observed significant improvements to the viability of agents with social support across a number of physicallychallenging conditions vs. conditions with no bonds. In many cases, the wider society also saw reduced stress (CT) levels and, as a result, improved viability. Comparing Experiments $2 \& 3$, we noted significant viability advantages for bonds with fixed affective bonds vs. variable bonds. In our model, we find that the buffering of the stress response via social support resulted in significant advantages to bonded, and in some cases unbonded, agent viability through behavioural adaptation and additional stress-reducing coping mechanisms. We propose that this type of behavioural and physiological adaptation via social interactions is a type of social allostasis. We complement our quantitative results with qualitative analysis of emergent interactions.

\section{“Tend-and-Befriend" vs. "Fight-or-Flight"}

We noted that for all bond combinations in Experiment 2, intra-bond Grooming increased as the world conditions became more challenging (Table 4). In the Seasonal and Extreme conditions, rather than become stressed and perform Aggression towards others, bonded agents would instead seek out social contact with their own affective bonds: performing Grooming, reducing CT, releasing OT and strengthening the bond between them (Figure 6, top right). This contributed to survival in future, more challenging situations (Table 2). We considered this an important behavioural response that facilitated viability in difficult conditions. When affective bond strength was fixed, we saw behaviours related to the "tend-and-befriend" hypothesis (Taylor, 2006), which proposes that, rather than looking for outlets of aggression, social individuals with strong relationships seek out positive social contact during times of stress. This behaviour is also seen to be related to individuals with higher levels of OT (Taylor, 2006), mirroring our results from Experiment 2 (Table 3).

In contrast, in experiments with variable bond strength and lower OT levels, we observed larger rates of intra-bond
Aggression as a coping behaviour for stress (Figures $5 \& 6$, bottom right). This behaviour then impacted affective bond strength and therefore survival rates of agents. Considering these results, we support the suggestion that additional context such as bond stability, partner identity and the degree of affiliation may affect the efficacy of social buffering effects (Kikusui et al., 2006), and therefore impact the different stress-reducing coping behaviours.

\section{Reconciliation is Hindered in Challenging Environments}

In some conditions in Experiment 3, we found that weaker affective bonds did not provide the hypothesised stressreducing effect when the world conditions were challenged (Seasonal and Extreme environments). Due to the lack of the stress-reducing effect from (weak) social support, (lowerranked) bonded partners were targeted for Aggression (Figure 5) by stressed agents, and intra-bond Aggression increased as a result (Table 5). This significantly reduced bond quality, and the ongoing stress caused by challenges from the Seasonal and Extreme environments limited the opportunity for Grooming between bond partners. This eventually resulted in a permanent loss of social support.

Again, we found that, despite social bond partners offering some "buffering" effect when bonds were strong (Experiment 2), these weakened bonds were susceptible to being lost beyond reconciliation during significantly challenging conditions. Therefore, in our model, the stress-reducing effects of social support was significantly affected by the environmental challenges and the strength of affective bonds between agents.

\section{Aggression Increases During Periods of Food Abundance}

In Extreme environments when bond strength was variable (Experiment 3), for agents in Bond Conditions B and C (when lower-ranked agents were bonded), we observed an increase in intra-bond Aggression when food availability was "good" (Phase 3, 5, 7, 9, Figure 5, bottom). Despite our prediction that aggression would decrease during "easier" physical periods, we saw that the magnitude of changes associated with the Extreme environment did not allow the agents to adapt to the constantly-changing conditions fast enough. As a result, the sudden shortage of food would increase group stress, which then carried over into the next phase (where food is abundant), increasing Aggression during these periods. This is not seen in Bond Condition A, or in conditions where the physical environment changes more gradually (Seasonal). In those conditions, we see $A g$ gression increase and decrease roughly in line with the food availability. In terms of the stress-reducing effects associated with social support in our model, we found that these effects were not one-size-fits-all, but impacted by the magnitude of the environmental challenge, the quality of affective 


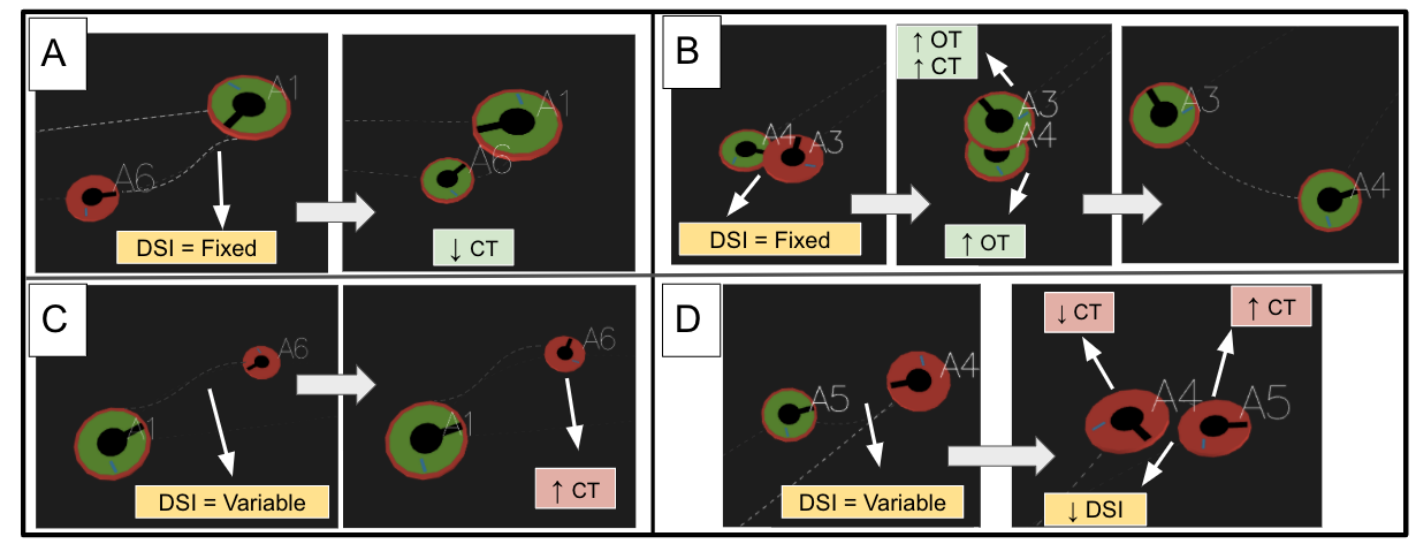

Figure 6: Screenshots of four different emergent social scenarios. Green agents indicate agents who are not stressed, red agents indicates agents who are stressed. A: Presence of social bond partner reduces stress levels. B: Tend-and-befriend behaviours during stressful situations when bonds are strong. C: A weak bond results in the bond partner becoming a source of stress, resulting in withdrawal. D: Intra-bond Aggression when bonds are weak, resulting in the bonded partner becoming stressed. DSI $=$ Dyadic Strength Index. CT $=$ Cortisol. OT $=$ Oxytocin .

bonds and social rank of bond partners.

\section{“Outsiders" can Damage Weaker Relationships}

In Experiment 3, we had noted that weak (variable) bonds had the potential to be indirectly and irreversibly damaged by outside social influences. Specifically, unbonded, higherranked agents (such as A2 or A3) would become stressed in physically-challenging periods. This resulted in Aggression towards lower-ranking agents (i.e., A4): increasing its stress level. For these lower-ranked agents, if their current bonds were already weakened, they would become aggressive towards their own (lower-ranking) bond partners. Rather than the "tend-and-befriend" behaviour that we described earlier (Figure 6, top right), these agents would perform Aggression towards their own bond partners (Figure 6, bottom right), reducing their affective bond strength. This loss of affective bond quality had an impact on the availability of future coping mechanisms and bond partner viability.

In these situations, when bonds were weak or if agents were experiencing higher levels of stress, socio-negative behaviours from higher-ranked members of society had a "trickle-down" effect on their interactions with bond partners. This loss of affective relationship resulted in subordinate bond partners withdrawing from interactions in the future (Figure 6, bottom left). In this scenario, an affective bond partner could go from being a source of social support to one of social stress. This highlighted the significance of the wider social context on the quality and maintenance of affective relationships. While affective bonds reduced stress in some contexts, this effect was impacted by significant environmental challenges and the wider social context.

\section{Conclusions}

In this paper, we have studied one aspect of the "social buffering" hypothesis, by investigating the effects of affective social support on the wellbeing and social interactions of individuals across a number of dynamic environmental conditions. We hypothesised that the presence of social bond partners would benefit the viability of our agents through a reduction of stress and behaviour adaptation. Using a simulated model, we have investigated these effects using a number of different affective bond combinations related to individual social rank, as well as both "fixed" and "variable" strengths of affective bonds. While we found some support for our hypothesis, we also found that the stress-reducing effects of social support were contextual, and that they were impacted by the degree of the wider social and environmental challenges, as well as the type of affective bond between agents. In many cases, we also found improvements in viability and reduced stress levels in agents without affective bonds, suggesting that the stress-reducing effects of social support may impact the wider social group in some conditions.

Extrapolating from our findings, we suggest that, for realworld societies, the stress-reducing effects of social support may not be universal, but that they may depend upon the relative environmental and social challenges, as well as the strength of affective relationships. As part of future work, we would like to see these findings of our model inspire new research in the study of related phenomena in biological agents.

\section{Acknowledgements}

Imran Khan is supported by a Ph.D. studentship from the University of Hertfordshire, UK. 


\section{References}

Andras, P. (2016). Social learning, environmental adversity and the evolution of cooperation. In Artificial Life Conference Proceedings 13, pages 290-297. MIT Press.

Antunes, R. F. and Magnenat-Thalmann, N. (2016). Bio-inspired virtual populations: adaptive behavior with affective feedback. In Proceedings of the 29th International Conference on Computer Animation and Social Agents, pages 101-110.

Ashby, W. R. (1954). Design for a Brain. Chapman \& Hall London.

Cañamero, L. D. (1997). Modeling motivations and emotions as a basis for intelligent behavior. In Proc. Agents'97, pages 148-155. ACM Press.

Cannon, W. B. (1929). Organization for physiological homeostasis. Physiological reviews, 9(3):399-431.

Covert III, A., McFetridge, S., and DeLord, E. (2014). Structured populations with limited resources exhibit higher rates of complex function evolution. In Artificial Life Conference Proceedings 14, pages 129-134. MIT Press.

DeVries, A. C., Glasper, E. R., and Detillion, C. E. (2003). Social modulation of stress responses. Physiology \& behavior, 79(3):399-407.

Heinrichs, M., Baumgartner, T., Kirschbaum, C., and Ehlert, U. (2003). Social support and oxytocin interact to suppress cortisol and subjective responses to psychosocial stress. Biological psychiatry, 54(12):1389-1398.

Hemelrijk, C. K., Kappeler, P. M., and Puga-Gonzalez, I. (2017). The self-organization of social complexity in group-living animals: Lessons from the DomWorld model. Elsevier Academic Press.

Hennessy, M. B., Kaiser, S., and Sachser, N. (2009). Social buffering of the stress response: diversity, mechanisms, and functions. Frontiers in neuroendocrinology, 30(4):470-482.

Holt-Lunstad, J., Smith, T. B., and Layton, J. B. (2010). Social relationships and mortality risk: a meta-analytic review. PLoS med, 7(7):e1000316.

Jolley, B. P., Borg, J. M., and Channon, A. (2016). Analysis of social learning strategies when discovering and maintaining behaviours inaccessible to incremental genetic evolution. In International Conference on Simulation of Adaptive Behavior, pages 293-304. Springer.

Khan, I., Lewis, M., and Cañamero, L. (2019). The effects of affective social bonds on the interactions and survival of simulated agents. In 2019 8th International Conference on Affective Computing and Intelligent Interaction Workshops and Demos (ACIIW), pages 374-380. IEEE.

Kikusui, T., Winslow, J. T., and Mori, Y. (2006). Social buffering: relief from stress and anxiety. Philosophical Transactions of the Royal Society B: Biological Sciences, 361(1476):22152228.

Levine, S. (2000). Influence of psychological variables on the activity of the hypothalamic-pituitary-adrenal axis. European journal of pharmacology, 405(1-3):149-160.
Lewis, M. and Cañamero, L. (2019). A robot model of stressinduced compulsive behavior. In Proc. 8th International Conference on Affective Computing \& Intelligent Interaction (ACII 2019). IEEE.

Lones, J., Lewis, M., and Cañamero, L. (2017). A hormone-driven epigenetic mechanism for adaptation in autonomous robots. IEEE Transactions on Cognitive and Developmental Systems, $10(2): 445-454$

Marriott, C. and Chebib, J. (2014). The effect of social learning on individual learning. In Artificial Life Conference Proceedings 14, pages 736-743. MIT Press

Miramontes, O., Boyer, D., and Ramos-Fernandez, G. (2016) Understanding fission-fusion dynamics in social animals through agent-based modelling. In Proceedings of the Artificial Life Conference 2016 13, pages 338-339. MIT Press.

Moulin-Frier, C., Puigbo, J.-Y., Arsiwalla, X. D., Sanchez-Fibla, M., and Verschure, P. F. (2017). Embodied artificial intelligence through distributed adaptive control: An integrated framework. In 2017 Joint IEEE International Conference on Development and Learning and Epigenetic Robotics (ICDLEpiRob), pages 324-330. IEEE.

Rajagopalan, P., Holekamp, K. E., and Miikkulainen, R. (2019). Factors that affect the evolution of complex cooperative behavior. In The 2018 Conference on Artificial Life: A Hybrid of the European Conference on Artificial Life (ECAL) and the International Conference on the Synthesis and Simulation of Living Systems (ALIFE), pages 333-340. MIT Press.

Santos, R., Marreiros, G., Ramos, C., Neves, J., and Bulas-Cruz, J. (2011). Personality, emotion, and mood in agent-based group decision making. IEEE Intelligent Systems, 26(6):58-66.

Schulkin, J. et al. (2004). Allostasis, homeostasis, and the costs of physiological adaptation. Cambridge University Press.

Sterling, P. (2004). Principles of allostasis: optimal design, predictive regulation, pathophysiology, and rational. Allostasis, homeostasis, and the costs of physiological adaptation, 17.

Taylor, S. E. (2006). Tend and befriend: Biobehavioral bases of affiliation under stress. Current directions in psychological science, 15(6):273-277.

Vouloutsi, V., Lallée, S., and Verschure, P. F. (2013). Modulating behaviors using allostatic control. In Conference on Biomimetic and Biohybrid Systems, pages 287-298. Springer

Wilensky, U. (1999). Netlogo. http: / / ccl . northwestern. edu/netlogo/. Center for Connected Learning and Computer-Based Modeling, Northwestern University, Evanston, IL.

Wittig, R. M., Crockford, C., Weltring, A., Langergraber, K. E., Deschner, T., and Zuberbühler, K. (2016). Social support reduces stress hormone levels in wild chimpanzees across stressful events and everyday affiliations. Nature communications, 7(1):1-8. 